\title{
Exposure to nickel from the metal equipment in the gym
}

\section{[version 1; peer review: 2 approved with reservations]}

\section{Denis Vinnikov (D1, Zhangir Tulekov1, Anar Dushpanova1, Zhanna Romanova1, Aleksandr Sokolov', Valeriya Sokolova1', Tair Nurpeisov²}

1 al-Farabi Kazakh National University, Almaty, 050040, Kazakhstan

${ }^{2}$ Scientific-Research Institute of Cardiology and Internal Diseases, Almaty, 050000, Kazakhstan

V1 First published: 17 Jan 2019, 8:68

https://doi.org/10.12688/f1000research.17791.1

Latest published: 17 Jan 2019, 8:68

https://doi.org/10.12688/f1000research.17791.1

\section{Abstract}

It remains unclear whether gym customers are exposed to any nickel from the metal equipment and if the exposure is associated with the duration of contact. Therefore, the aim of this study was to ascertain exposure to nickel measured through nickel concentration in the hair in those exercising in a fitness gym. We enrolled 100 amateur athletes in one of the gyms in Almaty, Kazakhstan (all men, median age 30 (interquartile range (IQR) 10) years), exercising from 2 to 7 days a week for 40 to 180 minutes and their age- and sex-matched controls who did not exercise. All subjects filled in the questionnaires on the exercising patterns, smoking and occupational exposure and then donated $0.25 \mathrm{~g}$ of head hair, in which nickel was measured using atomic absorption spectrophotometry. Hair nickel concentration ranged from 0 to $8.5 \mu \mathrm{g} / \mathrm{g}$ with notable left-skewness towards low concentrations in both groups. Hair nickel concentration was not associated with age, smoking or occupation, but was significantly lower in amateur athletes compared to controls (median 0 (IQR 0.5) vs. 0.9 (IQR 1.4) $\mu \mathrm{g} / \mathrm{g}$ ). More days a week in a gym, longer workout history, longer workout duration or supplements use did not increase the probability of being stratified in a high-exposure subgroup (defined as $75^{\text {th }}$ percentile of hair nickel concentration and higher); however, there were more smokers in a low-exposure group $(p<0.05)$. With the mixed pattern of exposure, gym goers may be unlikely exposed to more nickel from the metal equipment in the gym, however the exposure may depend on the specific alloy composition.

Keywords

gym, nickel, spectrophotometry, exposure

\section{Open Peer Review \\ Approval Status ? ? 2 \\ version 1 \\ 17 Jan 2019

$?$
view \\ $?$ \\ view \\ 1. Maria Jose Gonzalez-Munoz (iD), Universidad Alcalá de Henares, Alcalá de Henares, Spain \\ 2. Muhammad Abdul Qayyum, University of Education, Lahore, Pakistan \\ Any reports and responses or comments on the article can be found at the end of the article.}


Corresponding author: Denis Vinnikov (denisvinnikov@mail.ru)

Author roles: Vinnikov D: Conceptualization, Formal Analysis, Investigation, Supervision, Writing - Original Draft Preparation; Tulekov Z: Data Curation, Methodology, Writing - Review \& Editing; Dushpanova A: Data Curation, Formal Analysis, Methodology, Validation, Writing - Review \& Editing; Romanova Z: Data Curation, Formal Analysis, Project Administration, Resources, Writing - Review \& Editing; Sokolov A: Methodology, Resources, Supervision, Validation, Visualization, Writing - Review \& Editing; Sokolova V: Investigation, Methodology, Resources, Supervision, Visualization, Writing - Review \& Editing; Nurpeisov T: Data Curation, Formal Analysis, Project Administration, Validation, Writing - Review \& Editing

Competing interests: No competing interests were disclosed.

Grant information: The author(s) declared that no grants were involved in supporting this work.

Copyright: @ 2019 Vinnikov D et al. This is an open access article distributed under the terms of the Creative Commons Attribution License, which permits unrestricted use, distribution, and reproduction in any medium, provided the original work is properly cited.

How to cite this article: Vinnikov D, Tulekov Z, Dushpanova A et al. Exposure to nickel from the metal equipment in the gym [version 1; peer review: 2 approved with reservations] F1000Research 2019, 8:68 https://doi.org/10.12688/f1000research.17791.1

First published: 17 Jan 2019, 8:68 https://doi.org/10.12688/f1000research.17791.1 


\section{Introduction}

Exercising in a gym has become quite a prevalent leisure activity in adults, given significant benefit of regular exercising on cardiovascular health, mood and probably self-confidence. Little is known, however, about the adverse effects of attending the gym with the corresponding exposures. Gym goers may be exposed to a variety of chemicals inside gyms including metal bars and barbells. Those are usually produced of stainless steel, and the chemical composition of the latter may vary; however, selected metals in the steel, such as nickel, may be associated with adverse health effects in environmental and occupational studies. Thus, nickel is a known carcinogen ${ }^{1,2}$ and may also cause allergic dermatitis.

Very few studies, however, assess exposure of those exercising in the gyms to nickel. There is only one report with a small sample to show higher concentrations of nickel from the contact with bars compared to non-exercising individuals ${ }^{3}$. In this presentation, they found nickel both on the bars and the skin of exercising individuals using acid test. A case of allergic dermatitis was also reported in a regularly exercising individual ${ }^{4}$, but no studies with larger samples have approached the issue of nickel exposure and its adverse health effects in the gyms. Whether nickel found in the palm skin of gym customers may lead to higher nickel blood concentrations, therefore causing systemic effects, remains unknown. In professional sportsmen, nickel blood concentrations were found to be higher compared to controls ${ }^{5}$, and given that nutritional intake of nickel in these two groups did not differ, such findings should raise some concern as to whether such elevated concentration is associated with exposure in the gyms and has any negative impact on health.

With all that scarce evidence, it remains unclear whether gym customers are exposed to any nickel from the metal equipment and if the exposure is associated with the duration of contact. Therefore, the aim of this study was to ascertain exposure to nickel measured through nickel concentration in the hair in those exercising in a fitness gym compared to non-exercising controls.

\section{Methods}

\section{Ethical approval}

This study was approved by the Committee on Bioethics of al-Farabi Kazakh National University. All subjects in this study provided written informed consent to participate and donate head hair sample for nickel analysis.

\section{Recruitment and variables measured}

We enrolled 100 customers from two gyms in of one the popular chains in Almaty, Kazakhstan. Anyone willing to participate and regularly exercising in a gym could be included in the study. The only exclusion criterion was female sex, as there were very few women in the gym. Subjects were invited to participate by authors DV, ZhT or AD in a random fashion, thus, reducing selection bias. Data were collected in July and August 2018. Sample size calculation with a given statistical power did not seem feasible for this study, as we could not find any other similar analysis of this kind in the literature; therefore, we set the sample size of 100 subjects. We also enrolled sex- and age-matched controls who were their friends or acquaintances to ensure comparable lifestyle, eating habits and general interests to control for confounding. Controls should not have exercised in a gym for at least 2 years prior to the enrollment in the study. All subjects were asked to fill in a questionnaire ${ }^{6}$, which consisted of the demographic part, followed by detailed section in the exercising pattern, smoking, occupational exposure and the use of supplements. We asked the respondents how many days they normally attended the gym, what the duration of the usual workout was in minutes, how long was the gym exposure history, whether gloves were used in the gym, whether a contact allergy to metals in the gym was ever experienced, and whether any fitness tracker or supplement was used.

We then detailed smoking history with a series of questions and stratified all subjects into never, former or daily smokers and ascertained the number of smoked cigarettes a day along with the smoking durations in months or years. Occupational history section contained a series of questions whether a subject was a student at a time of the survey, had any employment in the office or had any occupational exposure with metal.

\section{Hair nickel concentration measurement}

We measured hair nickel concentrations in all subjects and treated the concentration as a marker of exposure to nickel. Hair (at least $0.25 \mathrm{~g}$ ) was cut in from the occipital. Hair samples were then washed using non-ionized surface-active solution, then acetone and then with non-ionized water. Weighed samples were treated with nitric acid $(67 \%)$ and hydrogen peroxide (30\%). Nickel concentrations in the samples were tested using atomic absorption spectrophotometry on Perkin Elmer AAnalyst 400 with HGA 900 (USA) and following officially approved protocol ${ }^{7}$. The lower limit of detection (LOD) in our analysis was $0.05 \mu \mathrm{g} / \mathrm{g}$.

\section{Statistical analysis}

Hair nickel concentrations were the primary outcomes in this analysis and compared in the main and control groups using non-parametric Mann-Whitney U-test, since all concentrations were left-skewed. Demographic attributes, smoking and occupational exposure were tested as predictors in bivariate models and compared between the groups. We used NCSS 12 (Utah, USA) for all computations. $\mathrm{P}<0.05$ was considered significant.

\section{Results}

\section{Hair nickel concentration}

Hair nickel concentration ranged from 0 to $8.5 \mu \mathrm{g} / \mathrm{g}$, with notable left-skewness towards low concentrations. Thus, $25^{\text {th }}$ percentile was $0 \mu \mathrm{g} / \mathrm{g} ; 50^{\text {th }}, 0.38 \mu \mathrm{g} / \mathrm{g}$; and $75^{\text {th }}, 1.22 \mu \mathrm{g} / \mathrm{g}$. A total of $56(28 \%)$ subjects showed concentrations below the limit of detection (LOD); $52 \%$ of amateur athletes and $4 \%$ of those in the control group had nickel levels below LOD $(\mathrm{p}<0.05)$. Hair nickel concentration was not associated with age, smoking or occupation, but was significantly lower in amateur athletes than controls (Table 1). Raw information of hair nickel concentration, in addition to all questionnaire answers, are available on $\mathrm{OSF}^{6}$. 
Variables in gym-goers

In the main group, the gym attendance frequency ranged from 2 to 7 days a week; however, most subjects did so 3 times a week (67\%). Workout duration ranged from 40 to 180 minutes; median, 90 (IQR 37.5) minutes. The overall gym exposure was from 1 month to 30 years, with the median 2 (IQR 4.4.) years. Only $17 \%$ of those in the gym used gloves for weightlifting on a regular basis, and $4 \%$ ever had dermatitis that they associated with the gym equipment use. A total of $16 \%$ use fitness tracker in the gym on a regular basis, and $56 \%$ use any sort of supplements to attain more visible results in the gym; there was no statistically significant correlation between these two variables.

\section{Analysis of hair nickel concentration}

When stratified by the $75^{\text {th }}$ percentile of nickel hair concentration $(0.505 \mu \mathrm{g} / \mathrm{g})$ into low- and high-exposure gym customers only, we found no difference in age, the number of workouts days a week, workout duration, overall exposure to gym equipment in years or supplement use (Table 2). Surprisingly, there were significantly more subjects wearing gloves, believed to protect the skin from contact with metal, in the high-exposure group. Similarly, the latter group had fewer smokers compared to those with lower nickel concentrations.

\section{Discussion}

To our knowledge, this is the first report on hair nickel concentrations in those attending the gym compared to controls, in which we could not confirm higher exposure to nickel in amateur athletes. Guided by the pilot presentations that exposure to metal equipment in the gym may result in greater nickel absorption, we compared hair nickel concentrations in regular exercisers compared to those abstaining from the gym, but found higher hair nickel concentrations in the latter group. We conclude that it was not dermal contact with metal equipment in the gym, but fewer smokers or specific nutritional habits in the gym goers group that could explain their lower hair nickel concentrations.

\section{Table 1. Demographic profile and nickel concentrations in the amateur athletes and controls.}

\begin{tabular}{|l|c|c|c|}
\hline Variables & Total & Gym & No gym \\
\hline N (\%) & $200(100)$ & $100(50)$ & $100(50)$ \\
\hline Age, years & $29(8.8)$ & $30(10)$ & $29(9)$ \\
\hline Never smokers, N (\%) & $84(42)$ & $33(33)$ & $51(51)^{\star}$ \\
\hline Daily smokers, N (\%) & $59(30)$ & $23(23)$ & $36(36)^{\star}$ \\
\hline Students, N (\%) & $49(25)$ & $17(17)$ & $32(32)$ \\
\hline Office staff, N (\%) & $89(45)$ & $48(48)$ & $41(41)$ \\
\hline $\begin{array}{l}\text { Occupational exposure to } \\
\text { metal, N (\%) }\end{array}$ & $5(3)$ & $4(4)$ & $1(1)$ \\
\hline Ni in hair, $\mu g / g$ & $0.38(1.22)$ & $0(0.5)$ & $0.9(1.4)^{\star}$ \\
\hline
\end{tabular}

${ }^{*} \mathrm{P}<0.05$, gym-goers compared to control.

Table 2. Selected predictors of higher hair nickel concentrations in gymgoers.

\begin{tabular}{|l|c|c|}
\hline Variables & High exposure & Low exposure \\
\hline N (\%) & $25(25)$ & $75(75)$ \\
\hline Age, years & $31(12)$ & $30(8)$ \\
\hline Days a week in a gym & $3(0.5)$ & $3(1)$ \\
\hline Workout duration, min & $90(45)$ & $100(30)$ \\
\hline Overall workout history, years & $2(4.4)$ & $2(4.4)$ \\
\hline Use of gloves, N (\%) & $12(48)$ & $5(7)^{\star}$ \\
\hline Supplements use, N (\%) & $16(64)$ & $40(53)$ \\
\hline Daily smoking, N (\%) & $6(24)$ & $53(71)^{\star}$ \\
\hline Occupational exposure to metals, N (\%) & $1(4)$ & $3(4)$ \\
\hline
\end{tabular}

${ }^{*} \mathrm{P}<0.05$, high-exposure compared to low-exposure group. 
The sources of nickel in the population may range from absorption to food, smoking, place of residence, lifestyle habits, such as exposure, to diverse occupational exposures. Despite some likelihood of exposure to nickel in those exercising in the gym, we could not find similar reports in the literature and could not compare the concentrations we found with other settings. However, there are plenty of other environmental and occupational publications with reported hair nickel concentrations.

The most surprising finding of this analysis was nickel concentration in controls. Although we deliberately matched controls with exercisers to ensure similar eating patterns, their hair nickel concentrations were quite high and even exceeded the concentrations in occupationally exposed industrial workers ${ }^{8}$. In order to allow for comparison between those exercising in the gym and controls, nutritional nickel consumption should be equal in both groups. Direct assessment of the amount of consumed nickel does not seem feasible in a regular setting; therefore, computational methods are often used in studies of athletes ${ }^{5}$. However, such methods yield more approximation than accuracy and therefore will lead to a notable exposure classification bias. Hence, in our study, we preferred to enroll controls from friends, matched for age and sex, to allow for comparable nickel consumption in the main group with controls.

The limitations of this analysis originate from its cross-sectional design. The overall sample size of 200 subjects may also limit statistical power. Another limitation is the use of matching rather than a detailed questionnaire on eating habits and computational method to ascertain food nickel consumption.
Finally, we could not obtain detailed information on the metal composition of the steel used for a particular brand of metal equipment in the chain of gym under study. Guided by anecdotal reports in non-professional literature, stainless steel for metal equipment in the gym is very likely produced of steel with some nickel content, but we could not confirm whether the given equipment had any nickel in it, either from the original documentation or, alternatively, using acid nickel testing.

To conclude, this pilot study of nickel exposure measured through hair nickel concentration in those contacting metal equipment in the gym failed to demonstrate greater hair nickel concentration in the latter compared to their non-exercising friends.

\section{Data availability}

Underlying data

Raw data for this study, including basic demographic information, answers to the questionnaire and hair nickel levels, are available on OSF. DOI: https://doi.org/10.17605/OSF.IO/RQJ3Z6.

\section{Extended data}

The questionnaire in the original (Russian) and in English are available on OSF. DOI: https://doi.org/10.17605/OSF.IO/ RQJ3Z6.

\section{Grant information}

The author(s) declared that no grants were involved in supporting this work.
1. Seilkop SK, Lightfoot NE, Berriault CJ, et al:: Respiratory cancer mortality and incidence in an updated cohort of Canadian nickel production workers. Arch Environ Occup Health. 2017; 72(4): 204-219. PubMed Abstract | Publisher Full Text

2. Grimsrud TK, Berge SR, Haldorsen T, et al.: Exposure to different forms of nickel and risk of lung cancer. Am J Epidemiol. 2002; 156(12): 1123-1132. PubMed Abstract | Publisher Full Text

3. Gumulka M, Matura M, Lidén $C$, et al.: Nickel exposure when working out in the gym. Acta Derm Venereol. 2015; 95(2): 247-249.

PubMed Abstract | Publisher Full Text

4. Ledon JA, Tosti A: CrossFit-Associated Allergic Contact Dermatitis. Dermatitis. 2017; 28(6): 368

PubMed Abstract | Publisher Full Text
5. Maynar M, Llerena F, Bartolomé I, et al.: Seric concentrations of copper, chromium, manganesum, nickel and selenium in aerobic, anaerobic and mixed professional sportsmen. J Int Soc Sports Nutr. 2018; 15: 8. PubMed Abstract | Publisher Full Text | Free Full Text

6. Vinnikov D: Hair nickel concentration in gym goers. 2019. http://www.doi.org/10.17605/OSF.IO/RQJ3Z

7. Iron, zinc, nickel, copper and chrome determination in hair using atomic absorbtion. Methodological recommentations. МУК 4.1.776-99. Ministry of Health of Russian Federation; 1999.

8. Vinnikov D, Semizhon S, Rybina T, et al:: Occupational exposure to metals and other elements in the tractor production. PloS One. 2018; 13(12): e0208932. PubMed Abstract | Publisher Full Text | Free Full Text 


\title{
Open Peer Review
}

\section{Current Peer Review Status: ? ?}

\section{Version 1}

Reviewer Report 28 September 2020

https://doi.org/10.5256/f1000research.19451.r71260

(C) 2020 Qayyum M. This is an open access peer review report distributed under the terms of the Creative Commons Attribution License, which permits unrestricted use, distribution, and reproduction in any medium, provided the original work is properly cited.

\section{Muhammad Abdul Qayyum \\ Department of Chemistry, University of Education, Lahore, Pakistan}

Reviewer comments: Reference to the Manuscript Number: F1 KR00CDE F1R-VER19451-R (end code), the authors describes $\mathrm{Ni}$ metal and its exposure to people in the gym and in relation to controls. The authors did good efforts to perform this kind of work which may be new one in their country/region. There are some minor revisions which ought to be correct before indexing.

\begin{abstract}
Line....measured using atomic absorption spectrophotometry.... (Should add).

The hair samples were digested/mineralized with the mixture of $\mathrm{HNO} 3$ and $\mathrm{H} 2 \mathrm{O} 2$ prior to analysis. Hair nickel concentration ranged from 0 to $8.5 \mu \mathrm{g} / \mathrm{g} . .$.
\end{abstract}

\section{Introduction}

The authors should write/add at least one paragraph which is comprised of hazards or toxicity of Nickel on human beings with references.

\section{Methods}

The authors should mention full name of city and country as in this sentence .... This study was approved by the Committee on Bioethics of al-Farabi Kazakh National University....... What this line explain........participate by authors DV, ZhT or AD in a random fashion? The authors indicate occupational exposure. I suggest authors should shown in name of occupational exposure as exposed by participants and also shown by duration in the table 1 . In statistical analysis $p<0.05, p$ should be in italic.

\section{Hair nickel concentration measurement}

The authors should write as followed or may your own style/words as well the method they adopted. This is a method for reference.

In the present study, scalp hair specimens (at least $0.25 \mathrm{~g}$ ) were collected from the occipital region of the head with a pair of stainless-steel scissors. The collected hair samples were put into small polyethylene bags, labelled with relevant codes and stored at room temperature until digestion and analysis of metals were performed. The hair samples were thoroughly washed to offer an 
accurate assessment of endogenous metal contents. Before washing, the samples were cut into small pieces (approximately $0.5 \mathrm{~cm}$ ) and mixed to make a representative sample. Afterwards, each hair sample was washed in series with $5 \%$ detergent solution, $0.5 \%$ Triton X-100 solution and deionized water. First of all, the scalp hair sample was taken in a conical flask containing $50 \mathrm{~mL}$ of $5 \%$ detergent solution and mixed well. The flask contents were then shaken on an auto-shaker at 320 vibrations per minute for about $30 \mathrm{~min}$. After leaving it at room temperature for at least $2 \mathrm{~h}$, it was washed with plentiful water. Then, $30 \mathrm{~mL}$ of non-ionic detergent Triton X-100 $(0.5 \% \mathrm{v} / \mathrm{v})$ solution was added to each flask and again placed on the auto-shaker for $30 \mathrm{~min}$. The samples were then washed with deionized water followed by drying in an electric oven overnight at $70{ }^{\circ} \mathrm{C}$ (Reference).

Similarly explain, the authors should write the complete process of digestion/mineralization e.g. heating, heating source and from what temperature used to complete process, duration of time as well as reference.

\section{Results}

Explain Table 1 completely in results section. For example and for reference.

Characteristics of the Study Subjects

The demographic parameters related to the stomach cancer patients and healthy donors are displayed in Table 1.

Stomach cancermalignancy was confirmed histopathologically along with clinical examination. The age of the stomach cancer patients ranged from 17 to 63 years with a mean value of 45 years while for healthy donors, it ranged from 15 to 65 years with a mean value of 42 years. Majority of the participants (>50\%) in both groups were vegetarians and 63\%of the patients and $64 \%$ of the healthy donors resided in the rural areas. More than $50 \%$ of the patients and healthy subjects were not addicted to tobacco (smoking). Most of the patients (68\%) were suffering from adenocarcinoma. Based on the division of histopathological stage, $32 \%$ of the patients were diagnosed at stage II, $25 \%$ at stage I, and $23 \%$ at stage III while $20 \%$ at stage IVof stomach cancer in the present study.

Also demonstrate table 2 in results more clearly.

In Table 1. Demographic profile and nickel concentrations in the amateur athletes and controls. Please correct.

Age, years $2959(8.8) 30(10) 29(9)$

\section{Summary}

Giving conclusions, the article has clear objective, approach is appropriate, However, the introduction does not provide any background on Ni toxicity/hazards/disease. The experiments and analyses performed with technical rigor to allow confidence in the results. But should be explain step by step. E.g., collection and processing of hair samples. Use of measuring unit is a good step and its name should be shown in the tables as well as in the context if needed. Some global points of view, at least to discuss the results, should be highlighted from relevant published reports. The variables shown in Tables are extracted conclusive information. Occupational exposure name with durations should be added in table 1. It seems number of samples are limited which we did not drive any clinically conclusion. However, after analyzing the data, results/tables are convincing especially elemental concentration. I suggest this manuscript is suitable for indexing.

\section{Is the work clearly and accurately presented and does it cite the current literature?}


Yes

Is the study design appropriate and is the work technically sound?

Yes

Are sufficient details of methods and analysis provided to allow replication by others? Partly

If applicable, is the statistical analysis and its interpretation appropriate?

Yes

Are all the source data underlying the results available to ensure full reproducibility? Yes

Are the conclusions drawn adequately supported by the results?

Yes

Competing Interests: No competing interests were disclosed.

Reviewer Expertise: Metal toxicology on carcinogenics/diseases, Phytochemistry,

I confirm that I have read this submission and believe that I have an appropriate level of expertise to confirm that it is of an acceptable scientific standard, however I have significant reservations, as outlined above.

Reviewer Report 23 September 2020

https://doi.org/10.5256/f1000research.19451.r69535

(c) 2020 Gonzalez-Munoz M. This is an open access peer review report distributed under the terms of the Creative Commons Attribution License, which permits unrestricted use, distribution, and reproduction in any medium, provided the original work is properly cited.

Maria Jose Gonzalez-Munoz

Department of Biochemistry, Universidad Alcalá de Henares, Alcalá de Henares, Spain

It is a very novel and interesting work, but a bit poor in its methodology and conclusions.

I send you the doubts:

Materials and methods

Subjects were invited to participate by authors DV, ZhT or AD in a random fashion, thus, reducing selection bias. I do not understand

\section{Determination of $\mathrm{Zn}$}

With what instrument was the sample collection carried out? Scissors? What material?

Was it taken into account if the hair was dyed or not? 
It could indicate the analytical parameters used for the determination of $\mathrm{Zn}$ by AAS.

The determinations were made in duplicate? Was a standard reference used? Which one?

\section{Results}

I don't understand the Tables, what do the numbers refer to? $i$

The authors send the data on the raw $\mathrm{Zn}$ levels in hair and the questionnaire used to another publication. I think it is not very comfortable to understand.

Is the work clearly and accurately presented and does it cite the current literature? Partly

Is the study design appropriate and is the work technically sound?

Yes

Are sufficient details of methods and analysis provided to allow replication by others? Partly

If applicable, is the statistical analysis and its interpretation appropriate? Partly

Are all the source data underlying the results available to ensure full reproducibility? No source data required

Are the conclusions drawn adequately supported by the results? Yes

Competing Interests: No competing interests were disclosed.

I confirm that I have read this submission and believe that I have an appropriate level of expertise to confirm that it is of an acceptable scientific standard, however I have significant reservations, as outlined above. 
The benefits of publishing with F1000Research:

- Your article is published within days, with no editorial bias

- You can publish traditional articles, null/negative results, case reports, data notes and more

- The peer review process is transparent and collaborative

- Your article is indexed in PubMed after passing peer review

- Dedicated customer support at every stage

For pre-submission enquiries, contact research@f1000.com 